Les dictionnaires bilingues des maîtres ou professeurs auteurs de manuels pédagogiques

\title{
Lexicographie bilingue et didactique : le Nuovo vocabolario comparativo delle lingue italiana e francese de Candido Ghiotti
}

\section{Annick Farina et Marie-France Merger}

\section{(2) OpenEdition}

Édition électronique

URL : https://journals.openedition.org/dhfles/3948

DOI : $10.4000 /$ dhfles.3948

ISSN : 2221-4038

Éditeur

Société Internationale pour l'Histoire du Français Langue Étrangère ou Seconde

Édition imprimée

Date de publication : 1 juin 2016

Pagination : 75-101

ISSN : 0992-7654

Référence électronique

Annick Farina et Marie-France Merger, « Lexicographie bilingue et didactique : le Nuovo vocabolario comparativo delle lingue italiana e francese de Candido Ghiotti », Documents pour l'histoire du français langue étrangère ou seconde [En ligne], 56 | 2016, mis en ligne le 04 septembre 2017, consulté le 05 avril 2023. URL : http://journals.openedition.org/dhfles/3948 ; DOI : https://doi.org/10.4000/dhfles. 3948

Ce document a été généré automatiquement le 5 avril 2023.

Tous droits réservés 


\title{
Lexicographie bilingue et didactique : le Nuovo vocabolario comparativo delle lingue italiana e francese de Candido Ghiotti
}

\author{
Annick Farina et Marie-France Merger
}

Dictionnaires, encyclopédies, grammaires sont donc à merveille les lieux où lire entre les lignes, où reconnaître, plus facilement qu'ailleurs, les conflits, les masquages des conflits, les clichés qui font l'album de famille d'une culture.

(Meschonnic 1992 : 16)

1 Dans le parcours des "maitres de langue " des siècles passés, on peut noter que la rédaction de dictionnaires bilingues comme outils d'accompagnement de leur enseignement de la langue allait souvent de pair avec la rédaction d'autres ouvrages à but didactique, des manuels d'apprentissage et des grammaires mais aussi, bien souvent, des textes ayant plus généralement la «culture » des locuteurs de la langue qu'ils enseignaient pour objet (ouvrages portant sur la littérature, sur l'histoire ou sur le patrimoine au sens large). Nous intéressant à la manière dont la diffusion de la culture de l'autre (la « civilisation étrangère ») a pu accompagner l'enseignement des langues étrangères et en particulier à la fonction qu'on a pu donner au dictionnaire bilingue dans cette diffusion, nous avons choisi d'examiner un ouvrage qui peut être considéré comme pionnier dans l'adaptation de la forme et du contenu du dictionnaire bilingue à des préoccupations didactiques, dans le contexte de l'enseignement du français langue étrangère en Italie : le Nuovo vocabolario comparativo delle lingue italiana e francese de Candido Ghiotti (1883).

2 Marie-France Merger (2003) a déjà souligné les motivations didactiques qui ont poussé Ghiotti à la rédaction d'un Vocabolario comparativo qu'il espérait voir devenir « une aide efficace pour l'enseignant et un bon guide pour l'apprenant", à l'instar de sa Grammatica ragionata della lingua francese (1868) qui reposait sur une méthode comparative que Ghiotti avait à cœur de voir appliquée dans les écoles italiennes. Nous montrerons combien l'engagement de Ghiotti dans la promotion d'une nouvelle 
manière d'aborder l'enseignement des langues étrangères en Italie et la priorité qu'il attribue, dans ce renouvellement didactique, aux outils utilisés par les élèves et par les enseignants se manifestent tant dans le contenu que dans la forme du Vocabolario. Après avoir mis en lumière la place du Vocabolario à l'intérieur de l'œuvre pédagogique de Ghiotti, Marie-France Merger illustrera l'adaptation de la microstructure de ce dictionnaire à des besoins didactiques linguistiques spécifiques, définis sur la base d'une réflexion sur l'utilisation de cet outil, pour ce qui concerne l'illustration des différences entre les deux systèmes décrits (français vs italien). Annick Farina s'attachera pour sa part à démontrer combien cette volonté d'instruire, et de le faire en partant de la différence, ne s'est pas seulement attachée à la mise en relation de mots et de structures des deux langues mais s'applique aussi, à l'intérieur du Vocabolario comparativo, à des aspects concernant les cultures véhiculées par ces langues. Elle tentera ainsi de rendre compte plus particulièrement de la « mission pédagogique » à laquelle a pu participer Ghiotti en inscrivant son Vocabolario dans un projet plus large de diffusion d'une vision de la civilisation française édifiée en modèle dans l'Italie postunitaire.

\section{L'œuvre de Ghiotti}

3 En consultant son dossier aux Archives de la Pubblica Istruzione à Rome, nous apprenons que Ghiotti est né à Turin en 1841 ; après avoir suivi un « Cours complet », il obtient un diplôme correspondant au baccalauréat de l'institut technique, puis un brevet l'habilitant à l'enseignement du français et de l'anglais. Ses débuts dans l'enseignement sont placés «sous le signe de la précarité, de la mobilité territoriale et de la pluralité des disciplines enseignées » (Merger 2005 : 75) : il est très jeune quand il débute sa carrière à Alessandria en 1859 dans une Scuola Tecnica, puis il enseignera à Turin, à Pesaro dans les Marches pour revenir à Alessandria (1864-1871). En 1872, il est muté à Frosinone (Latium), toujours dans une Scuola Tecnica, et ce n'est qu'en 1880 qu'il sera nommé professeur titulaire de français et directeur de cette école. Les renseignements sur sa carrière s'arrêtent là, néanmoins la préface de son dictionnaire bilingue nous indique qu'en août 1883, il était professeur à la Regia Scuola Tecnica Germano Sommeiller de Turin; il en est devenu le directeur et c'est là qu'il a fini sa carrière. Il est mort en 1915.

4 Le nom de Ghiotti est inséparable de sa grammaire et de ses dictionnaires, mais il ne s'est pas limité à ces ouvrages. En effet, en 1865, il publie une chrestomathie, Crestomazia tecnica francese, proposta agli alunni dei corsi tecnici, dont les rééditions seront nombreuses, puis une refonte des dialogues de Morand, Le nouveau Morand, des anthologies, notamment Le nouveau Noël et de La Place qui a eu au moins neuf éditions. Avec son collègue G. Dogliani, il publie de nombreuses anthologies, un manuel de correspondance commerciale, des exercices de conversation et des livres de lecture.

\section{La longue vie de la Grammatica ragionata della lingua francese}

5 Lorsque Ghiotti publie, en 1868, sa Grammatica ragionata della lingua francese, l'Unité italienne est réalisée depuis peu et la Péninsule est un pays encore très dialectophone malgré la loi Casati qui a organisé tout le système scolaire, notamment l'enseignement secondaire. À l'époque les écoles techniques et les instituts techniques étaient les seuls 
établissements scolaires où l'on enseignait les langues vivantes, le français y était obligatoire et, comme deuxième langue, l'allemand ou l'anglais. Cette grammaire porte un sous-titre sans équivoque: "Nouveau cours de leçons théoriques et pratiques dictées avec la comparaison avec l'italien et avec l'analyse philologique ", que l'auteur reprend dans sa Préface en illustrant sa démarche qu'il qualifie de «metodo filologico comparato ». D'après lui, pour enseigner le français, il faut partir de la connaissance de la langue maternelle, l'italien. Il faut exploiter les ressources qu'offre la parenté de l'italien et du français, des langues proches par leur origine et par leur histoire (Ghiotti 1875 : III). Il s'agit d'une méthode déductive fondée sur le raisonnement - le contraire des méthodes «pratiques » (Pellandra $2004: 85$ ) d'Ahn et d'ollendorff - une méthode qui fait appel à l'intelligence des élèves, qui forme l'esprit comme le faisait l'étude des langues classiques, le latin et le grec. Le français et son enseignement sont ainsi élevés au rang des langues nobles, le français devient une discipline scolaire.

«La méthode 'classique' de Ghiotti était centrée sur l'écrit et par écrit on entendait la traduction dans la langue étrangère " (Pellandra 2004: 102), ce n'est donc pas un hasard si Ghiotti introduit deux traductions en français, deux thèmes à la fin de chaque leçon de sa grammaire. Le premier est une traduction interlinéaire où sont introduits les mots ne se rapportant pas aux règles exposées auparavant. "Le second thème est proposé sans la partie matérielle du lexique et cela pour habituer les élèves à la recherche, au choix logique des mots, car l'utilisation du dictionnaire a aussi ses difficultés » (Ghiotti $1875:$ [VII]), qu'il faut surmonter par la pratique.

7 La grammaire de Ghiotti et son résumé (Compendio) seront de véritables best-sellers, et seront les ouvrages les plus répandus au tournant du siècle et même au-delà. C. Pellandra nous signale en effet que l'ouvrage est encore publié en 1945 (alors que Ghiotti est mort depuis trente ans) sous le titre Compendio della grammatica ragionata della lingua francese riveduta dal Prof. Silvio Pons et qu'on est arrivé au $424^{\mathrm{e}}$ millier d'exemplaires (ibid. : 98).

\section{Le Nuovo Dizionario comparativo delle lingue italiana e francese}

8 C'est en 1883, «après sept ans de travail patient et très fatigant ${ }^{1}$ » que Ghiotti fait paraitre chez Bellardi et Appiotti le premier volume de son dictionnaire bilingue (italien-français), portant le titre suivant: Nuovo Dizionario comparativo delle lingue italiana e francese. Ce volume comprend XXIII pages liminaires et 1124 pages de nomenclature. La numérotation se poursuivra dans la seconde partie (français-italien) qui sera publiée un an plus tard; le dictionnaire comptera ainsi 2352 pages. Quelques années plus tard, en 1890, Ghiotti publie la seconde édition mais avec un titre légèrement différent: Nuovo Vocabolario comparativo delle lingue italiana e francese; dans la préface à la nouvelle édition "stéréotype ", l'auteur signale qu'il a ajouté quelques milliers de nouvelles entrées et que son ouvrage offre donc une comparaison plus précise, plus complète entre les deux langues. Le premier volume compte XXIV pages liminaires et 1572 pages de nomenclature ; le second volume, publié en 1892, ne se compose que de 1326 pages. Les éditions «stéréotypes " comportant toutes le même nombre de pages vont se succéder à partir de 1895, une fois que Ghiotti publiera chez Petrini à Turin.

9 Nous avons vu que Ghiotti privilégie l'écrit, et par écrit il entend la traduction en français (le thème), c'est pourquoi dans la Préface de 1883, il explique sa démarche 
"pédagogique » : pour aider les élèves à discerner les différentes acceptions d'un mot dans la série d'équivalents proposés par les dictionnaires scolaires, une série qui se présente comme «un enchevêtrement inextricable ${ }^{2}$ " (Ghiotti 1890: VI), il faut expliquer toutes les acceptions les moins connues du même mot, comme nous le montre l'exemple suivant tiré de son dictionnaire :

ÉTERE sm. ( $p$. la parte più sottile e più sublime dell'aria) éther (pron. etèr in tutte le sue accez. : l'air le plus pur) \| (poet., p. etera, aria ; cielo) l'éther, l'air ; le ciel \| (t. fis.) éther \| (t. chim.) éther (liquide très volatil qu'on obtient par la distillation d'un acide mêlé avec de l'alcool) : etere solforico, éther sulfurique.

10 Nous voyons que les gloses explicatives sont développées et que les différences de sens sont données dans la langue de l'entrée (l'italien) si elles précèdent l'équivalent et, en français, si elles le suivent. Dans le second volume, elles suivent l'équivalent, elles sont donc en italien, ce qui n'est pas surprenant puisque l'auteur s'adresse surtout aux élèves italiens.

Ghiotti fait suivre sa préface d'un petit traité de prononciation où il expose les règles générales pour bien prononcer les mots; il s'agit d'un résumé de celui qui se trouve dans sa grammaire que nous avons déjà évoquée. Pour les mots français difficiles ou pour ceux dont la prononciation ne suit pas les règles générales, l'auteur signale la prononciation exacte OSIRIDE (mitol.) Osiris (pron. la s finale), ou bien il transcrit graphiquement la prononciation: «PIRRO (stor.) Pyrrhus (pron. pirrüss')», « BUENOSAYRES (geog.) Buenos-Ayres (pron. buenòss-a-irèss' [...]) » (sic).

Il en est de même pour l'orthographe : l'auteur signale l'orthographe qu'il faut adopter comme dans « EREMİTA sm. hermite, ermite (questa è ortografia preferibile) [...]", et nous voyons qu'il préfère l'orthographe «isoscèle » à celle d'aujourd'hui. Ghiotti signale également les mots italiens dont l'orthographe n'est pas correcte comme « INCIELARE

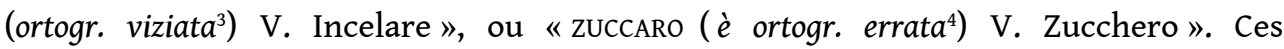
indications ne sont pas inutiles car à l'époque l'italien n'est pas parlé par tous, de là un des soucis constants des professeurs de langues vivantes : les élèves doivent apprendre une langue étrangère mais avant tout ils doivent parler un italien correct, la langue nationale étant prioritaire.

Ainsi ce dictionnaire est truffé de remarques axiologiques du type "voce dell'uso comune, ma non italiana ${ }^{5}$ » (Revolver); «è brutta voce da evitarsi ${ }^{6}$ " (Preludiare pour Preludere), etc. Ces remarques sont suivies de la forme correcte précédée des futurs injonctifs : « dirai meglio, dirai meglio però, adoprerai di preferenza queste voci, dirai in buon italiano ${ }^{7}$ ", et même du futur «dirai » (tu diras), "sans le bémol 'meglio'" (Merger 2007 : 61). Nous retrouvons ces marques axiologiques lorsque Ghiotti introduit les anglicismes et surtout les gallicismes, beaucoup plus nombreux à l'époque. Ses remarques peuvent aller d'un simple « è francesismo » (PIROETTA ${ }^{8}$ ou BONETTO ${ }^{\circ}$ ), jusqu'au jugement plus sévère avec la condamnation du "sguaiato francesismo " (gallicisme grossier) pour BUFFÉ ${ }^{10}$. Toutes ces formes sont suivies du mot correct que l'usager doit utiliser, ce qui fait de ce dictionnaire un ouvrage normatif.

\section{Le Vocabolario scolastico delle lingue italiana e francese}

À la demande des professeurs de français qui se plaignent de l'impossibilité d'utiliser le Comparativo qui s'adresse plutôt aux professeurs qu'aux élèves du fait de son format et de son prix trop élevé, Ghiotti publie son Vocabolario scolastico, chez Petrini en 1889, et 
c'est avec ce dictionnaire bilingue en un volume qu'il s'inscrit dans la lignée des dictionnaires scolaires où il occupera une place de première importance.

Ce dictionnaire que l'auteur présente comme un abrégé de son Comparativo compte 18 pages liminaires et 1341 pages de nomenclature. Son succès sera éclatant puisque, en 1902, la $25^{\circ}$ édition sera entièrement refaite et comptera 1598 pages. C'est cette édition qui servira aux éditions et aux réimpressions successives qui seront très nombreuses et non datées; le nombre de pages restera inchangé jusqu'à la nouvelle édition d'Antonio Chanoux en 1928. Contrairement au Vocabolario comparativo, il ne contient pas de précis grammatical, ni de traité de prononciation. La nomenclature est évidemment plus limitée, de même les explications, notamment celles qui donnaient un caractère encyclopédique au précédent, sont moins développées. Néanmoins les objectifs sont les mêmes, c'est toujours un dictionnaire normatif, et nous retrouvons donc les mêmes remarques indiquant les erreurs que l'élève doit éviter en italien comme en français. En outre, comme il s'agit d'un dictionnaire scolaire, la visée moralisante est présente et les mots considérés comme étant tabous sont éliminés de la nomenclature comme le mot «ZÀNZERO " qui était présent dans le Vocabolario Comparativo: "ZÀNZERO sm. (voce oscen ${ }^{11}$ ) bardache » mais qui a été supprimé dans le Scolastico (cf. également Merger $2003: 173)$.

Ce Vocabolario scolastico est devenu un 'outil à thèmes', c'est-à-dire un instrument utile pour rédiger, composer et traduire en français, et non plus un outil pour lire des textes en langue étrangère. Mais nous pouvons affirmer, avec J. Lillo, qu'« aucun auteur n'a eu le succès ni la longévité de Ghiotti» (Lillo 2006: 226), puisque son nom est resté pendant un siècle ou presque dans le catalogue de la maison d'édition Petrini, grâce aux révisions et aux rééditions de Chanoux et de Cumino.

\section{La « civilisation française " dans le Nuovo vocabolario comparativo delle lingue italiana e francese de Candido Ghiotti}

Christian Puren (1988: $114 \mathrm{sq}$ ) présente l'introduction de la "méthode directe », qui s'impose dans l'enseignement secondaire français par les instructions de 1890, 1901 et 1902, comme marquant un tournant dans l'introduction de la civilisation dans l'enseignement des langues, qui se révèle dès le début $\mathrm{du} \mathrm{XX}^{\mathrm{e}}$ siècle dans un renouvellement tant des activités proposées aux élèves que des types de supports proposés en classe :

Ce qui frappe l'historien dans ces instructions de 1901 et 1902, c'est leur très large ouverture méthodologique et thématique : variété des activités proposées en classe (explications de textes, lectures collectives, exposés, résumés de lectures individuelles, discussions,...) ; variété des supports didactiques utilisables (outre les extraits littéraires et les sommaires, dialogues et autres textes fabriqués, l'instruction de 1902 recommande l'usage « de cartes géographiques, de vues, de journaux, de revues, de collections pour bibliothèques scolaires, etc. »; variété des aspects de la civilisation étrangère à présenter [...]; variété des approches de la civilisation enfin - à la fois historique, géographique, culturelle, économique, anthropologique,... -, avec déjà cette idée, que reprendra l'instruction de 1908 parce qu'elle est dans la logique de l'approche directe, de « faire vivre » dans une certaine mesure aux élèves la culture étrangère "de l'intérieur ", en leur faisant lire les 
poésies, les contes, les romans, les anecdotes et récits historiques que lisent les enfants étrangers de leur âge. (Puren 1988 : 118) l'enseignement $d u$ français, et amène à modifier des manuels dans lesquels sont intégrés des textes qui devaient permettre « de faire connaître ce peuple dans tout ce qu'il a de caractéristique : ses gloires et ses malheurs, sa langue, sa littérature classique et populaire» (Gaetano Darchini 1894 cité par Minerva 2002: 125). Ghiotti participe évidemment à ce renouveau didactique, et propose de répondre à ces nouvelles nécessités par l'introduction d'informations culturelles dans son dictionnaire. C'est tout au moins ce qui ressort clairement de la « préface à la nouvelle édition » introduite en 1890 dans le Vocabolario comparativo :

[Les éléments suivants] contribuent à rendre l'Ouvrage meilleur et plus complet, relativement aux exigences de nos collèges et lycées : toute la partie qui se réfère à l'histoire, à la mythologie, à la géographie a été insérée, en suivant l'ordre alphabétique, dans le texte de la nomenclature générale des deux langues, avec les nouvelles historiques, géographiques, statistiques qui l'illustrent; les explications relatives aux termes techniques des sciences, des arts, des métiers, des industries; la prononciation des noms propres, celle des entrées à la prononciation irrégulière ou douteuse ou peu connue; les formes des verbes irréguliers; les observations grammaticales relatives à des changements de genre que connaissent certains noms selon leur acception, et enfin la plus large part a été donnée à la correction de vrais gallicismes et des formes erronées, avec renvoi aux formes de la bonne langue qui peuvent les remplacer tout aussi efficacement, et certainement avec une plus grande pureté d'élocution. (Ghiotti [1890] 1910 : IX [ma traduction] ${ }^{12}$ )

L'augmentation de la nomenclature du Vocabolario par l'intégration, en particulier, d'un grand nombre de noms propres mais aussi de termes techniques et l'introduction d'informations pensées en fonction de ce nouvel apport centré sur le «monde " plus que sur la langue en elle-même (les «nouvelles historiques, géographiques, statistiques ", par exemple) sont particulièrement importantes. Elles permettent de mesurer combien l'évolution des pratiques pédagogiques de l'époque de Ghiotti a pu avoir un effet sur la structure de ce dictionnaire et sur sa teneur idéologique - la "vision du monde» dont il se fait vecteur et qui est celle que promouvait l'école italienne post-Risorgimento. Cette modification initiée par Ghiotti ne sera pas sans effet sur les microstructures des dictionnaires bilingues français-italien rédigés par ses successeurs et sur la fonction primordiale que ces dictionnaires ont acquise comme outils pour l'enseignement des langues étrangères. Le contenu culturel du Vocabolario ne s'arrête cependant pas à ce que Ghiotti a introduit dans cette refonte de 1890 ni aux seuls articles qui concernent «l'histoire, la mythologie et la géographie ». Pour pouvoir analyser sa texture et sa teneur, c'est l'ensemble de l'ouvrage qu'il faut observer, en distinguant les différents lieux où s'intègre l'information culturelle. Plus précisément, afin d'illustrer l'engagement de Ghiotti, professeur de français en Italie à la fin du XIX ${ }^{\mathrm{e}}$ siècle, j'observerai le mode d'insertion de la culture française dans la partie françaisitalien du Vocabolario (volume 2). 


\section{La « vision du monde " promue par Ghiotti telle qu'elle se dessine dans le Vocabolario : les modes d'insertion de l'information culturelle dans la micro et macrostructure du dictionnaire}

Le dictionnaire s'impose généralement au regard des lecteurs comme une nomenclature exhaustive représentant une totalité - une langue décomposée en unités - et des informations strictement linguistiques - et donc savantes et objectives qui ne laissent que peu de place à des choix volontaires de la part du lexicographe ou à l'expression de ses idées et de sa personnalité.

21 Le Vocabolario comparativo, comme l'a déjà montré Merger (2007:64) appartient à une tradition de dictionnaires dans lesquels la présence de ce qu'elle appelle le «moi locuteur » peut s'exprimer dans des assertions et injonctions personnelles intégrées dans les articles lexicographiques ${ }^{13}$. Ces interventions marquent des prises de position de Ghiotti principalement sur le bon usage des deux langues qu'il décrit mais aussi, parfois, sur leurs référents ${ }^{14}$. Si l'analyse de ces assertions peut permettre de comprendre certaines des opinions de Ghiotti, elles ne constituent cependant que l'un des modes d'insertion d'une information culturelle transmise par l'auteur, volontairement ou à son insu, sur laquelle portera plus précisément notre travail.

Un dictionnaire traduit profondément son époque, à l'insu de ses rédacteurs, et c'est bien lexiculturellement qu'on le consulte, en prenant pour allant de soi ce qui n'est que l'application momentanée de points de vue qui ne cesseront d'évoluer (Pruvost 2009 : 142-143).

Comme tout dictionnaire d'hier et d'aujourd'hui le Vocabolario peut donner lieu à une lecture « lexiculturelle » au-delà des interventions manifestes de son auteur. J'ai déjà souligné que la décision de Ghiotti d'introduire des noms propres dans sa nomenclature (alors qu'ils étaient jusqu'alors relégués aux marges des dictionnaires bilingues à l'intérieur de listes) correspond à une volonté de s'adapter aux nécessités didactiques de son époque, en particulier à l'introduction de la civilisation dans l'enseignement des langues étrangères. Mais sa nomenclature, non exhaustive évidemment, révèle des choix qui n'ont rien d'anodin, tant pour les noms propres que pour les noms communs et en particulier pour ceux qui se réfèrent à des réalités françaises non partagées avec le commun des locuteurs de l'italien (les anthroponymes relatifs à des personnalités françaises, les toponymes et les realia français) : en choisissant d'introduire certains mots et d'en ignorer d'autres, Ghiotti manifeste son intérêt ou son indifférence pour tel aspect plutôt que tel autre de la culture française, en fonction de goûts qui lui sont parfois particuliers mais qui sont aussi et principalement ceux de son époque, et qui correspondent au contenu d'un enseignement qu'il souhaite promouvoir par la rédaction de son dictionnaire.

Pour ce qui concerne la microstructure, les différentes catégories d'articles ont un traitement différent: pour les noms propres, les entrées sont parfois suivies d'une indication sur leur prononciation comme indiqué dans la préface déjà citée, d'une abréviation de type thématique («stor.» pour histoire pour les anthroponymes, "geog.» pour les toponymes), suivent la forme italienne correspondante ${ }^{15}$ et des informations encyclopédiques en italien fournies à l'intérieur de parenthèses ${ }^{16}$. Ces informations correspondent aux exercices scolaires et aux méthodes d'enseignement de l'époque : apprentissage essentiellement chronologique de l'histoire représenté par la forte présence de dates dans le dictionnaire, point de vue anecdotique qui caractérise 
un enseignement visant à éveiller l'intérêt des élèves ${ }^{17}$, réduction de la géographie à un appendice de l'histoire dans des informations concernant les seules divisions politiques et administratives, etc.

Le traitement des noms communs révèle principalement l'approche comparatiste de Ghiotti pour ce qui concerne l'enseignement du français aux élèves italiens et son purisme linguistique relié à une volonté d'imposer une norme unificatrice d'italien dans un pays encore fortement marqué par les différences dialectales. C'est la raison pour laquelle seul le traitement des realia m'a semblé digne d'une attention particulière pour illustrer la vision de la culture française et de la différence culturelle entre France et Italie : dans les articles relatifs à ce type de mots, une simple traduction, lorsqu'elle existe, n'est généralement pas suffisante et Ghiotti définit donc ces mots pour son lecteur italien.

\section{Les choix de nomenclature : I'Histoire de France au premier plan.}

Une observation attentive de l'ensemble des entrées relatives à des noms propres et aux realia ( $c f$. plus loin) intégrés dans le deuxième volume (du français vers l'italien) du Vocabolario atteste l'importance primordiale donnée par Ghiotti à des thèmes qui accompagnaient l'enseignement de l'histoire par rapport à d'autres thèmes qui participent de la culture en général et de la culture française en particulier (littérature, arts, etc. $)^{18}$.

Si l'on regarde la distribution des fonctions associées à des anthroponymes qui apparaissent dans le dictionnaire de Ghiotti, on remarque la préférence marquée qu'il peut avoir pour des personnages qui ont eu des fonctions au niveau de la politique, de l'économie et de l'armée par rapport aux grands artistes ou scientifiques. Le schéma suivant illustre cette distribution à l'intérieur de l'ouvrage :

\section{Distribution des fonctions exercées par les personnalités citées dans le Vocabolario}

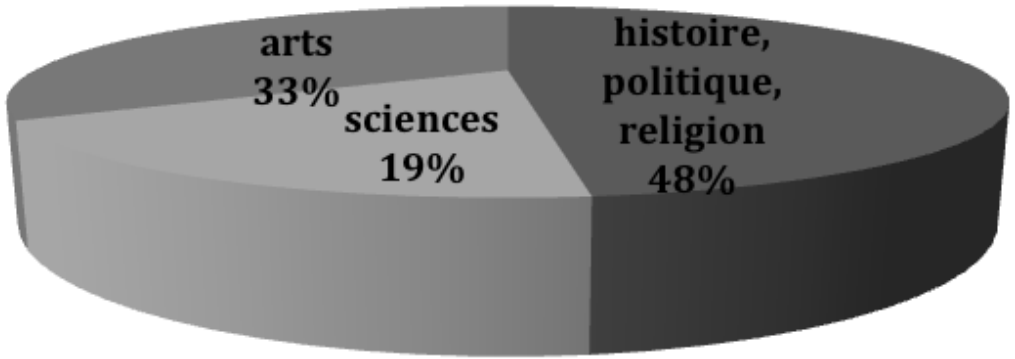

Dans les personnalités "historiques, politiques et religieuses », les personnalités les plus représentées sont les maréchaux, suivis de près par les généraux et les ministres (une cinquantaine chacun), puis par les «hommes d'État» et les évêques. Dans la catégorie des artistes, près de $80 \%$ sont des artistes « de plume » (150 environ) tandis que les peintres (surtout), les sculpteurs et les architectes se partagent les $20 \%$ restants, avec une poignée de compositeurs. Quant aux "scientifiques ", la moitié sont des philosophes et des historiens qui pourraient, de fait, être recensés dans la première 
catégorie ou la deuxième selon les cas. Pour les astronomes, mathématiciens, ingénieurs, physiciens et géographes on les trouve tous dans une même proportion dans l'ouvrage (entre 6 et 8 personnes citées).

L'intérêt de Ghiotti ne se porte pas non plus dans une même mesure sur toutes les périodes historiques : comme le révèle clairement le graphique suivant basé sur les dates trouvées dans la microstructure du Vocabolario, c'est le XVIII e siècle qui est le plus représenté dans ce dictionnaire :

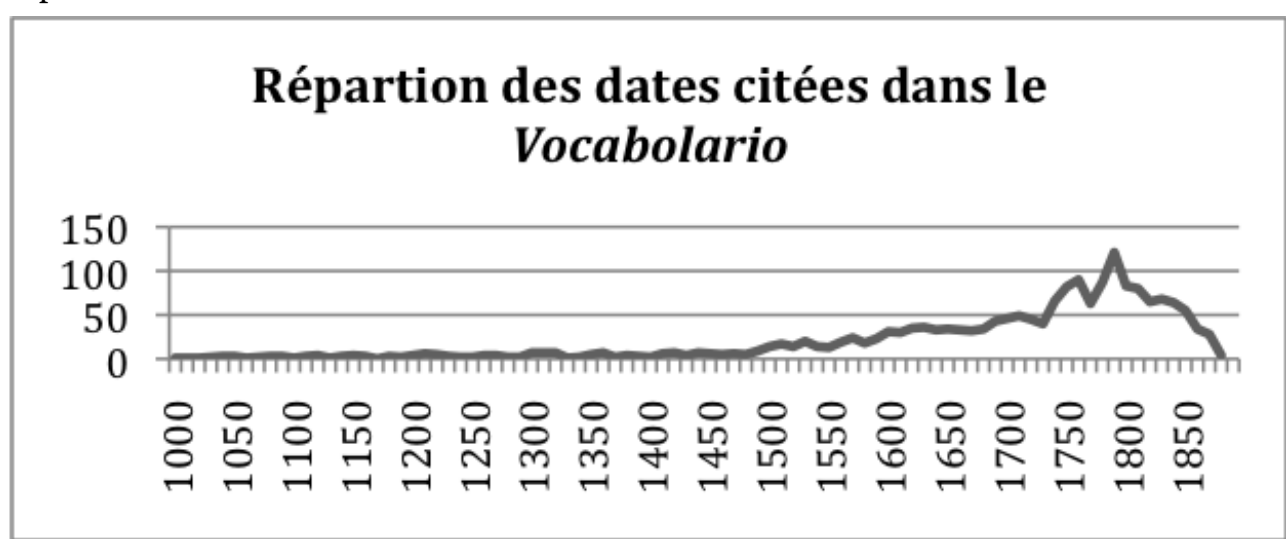

Une analyse plus attentive de ces dates, en distinguant les dates de naissance et celles de mort des personnalités citées et les autres dates qui apparaissent dans les articles (événements historiques), nous permet de repérer la période révolutionnaire et celle qui succède immédiatement à la Révolution française comme les plus présentes dans le dictionnaire. Ce ne sont pas en effet les nouveau-nés qui intéressent Ghiotti : les dates de naissance les plus représentées, autour de 1760, sont celles de personnalités dont la vie d'adulte se situe à la fin du XVIII ${ }^{e}$ siècle et au début du XIX ${ }^{\mathrm{e}}$. Ce qui est confirmé par la grande présence de dates de décès aux alentours de $1840-1850^{19}$.

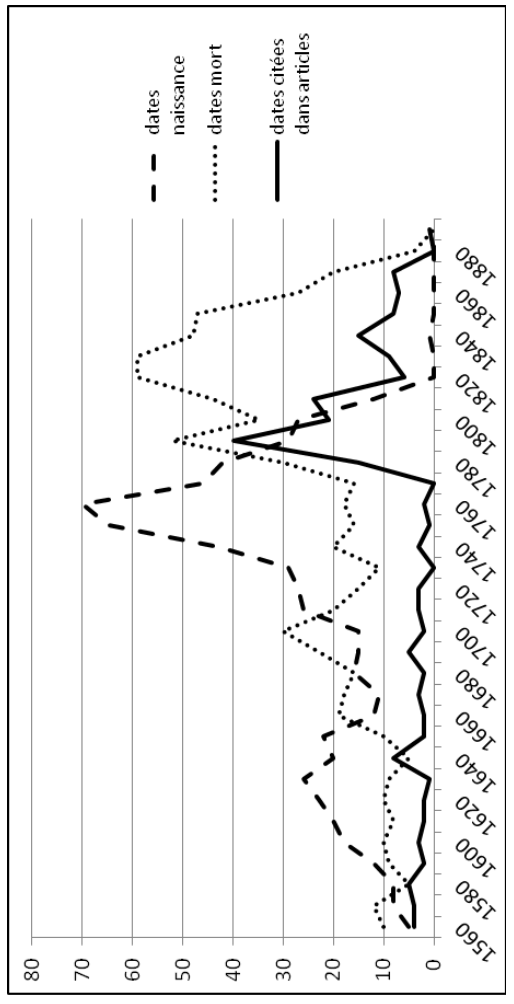




\section{Les realia dans le Vocabolario : les mots d'une nation} traitement dans les dictionnaires monolingues et bilingues d'aujourd'hui ${ }^{20}$. Ces mots se réfèrent à des objets typiques d'un lieu donné, souvent liés à des particularités historico-sociales ou administratives, à des croyances, des éléments du folklore (musiques, gastronomie, ...) et nécessitent pour cette raison une explication (glose ou note culturelle) décrivant le plus souvent non seulement le référent du mot mais plus largement le contexte culturel qui l'accompagne. Cette insertion d'informations de type encyclopédique se référant au "monde de l'autre » est particulièrement intéressante pour aborder le contenu de civilisation du dictionnaire de Ghiotti. Pour juger des choix de Ghiotti, je partirai cependant d'abord des éléments qu'il n'a pas choisi d'intégrer dans son dictionnaire, qui se réfèrent à des aspects de la culture française qui ne l'intéressent pas ou qu'il estime n'avoir aucune valeur dans la perspective de l'enseignement du français en Italie.

31 La première grande absence dans le Vocabolario concerne les réalités régionales françaises: alors qu'une recherche dans le texte complet du dictionnaire des mots « francese » ou « Francia » nous a permis de trouver nombre de gloses qui décrivent des réalités françaises que nous citerons plus loin, une recherche de noms de villes ou de régions françaises et des adjectifs dérivés de ses toponymes ne nous portent qu'à des articles relatifs à ces toponymes. Dans sa préface, Ghiotti cite le Littré comme sa référence pour la langue française. J’ai donc cherché dans le Littré des realia se référant à des spécialités régionales et ai trouvé par exemple pour la Bretagne un instrument de musique, le bignou, un gâteau, le cuigne (kouign aman), des fromages pour le Lyonnais (le cabrillon) et pour les Vosges (le géromé), mais aussi les tripoux («nom qu'on donne dans les Vosges à des terrains communaux divisés par lots »), etc. Aucun de ces mots ni de la vingtaine d'autres que j'ai recherchés dans le Vocabolario n'apparaît et toutes les realia françaises trouvées se réfèrent exclusivement à des objets connus et/ou utilisés sur tout le territoire français ${ }^{21}$. L'image d'une France unie autour d'une culture partagée va évidemment dans le sens du modèle de citoyen dont les institutions du jeune État italien unifié, et l'école italienne en particulier, sont les promotrices. À côté de cet effacement des particularités des régions françaises, ne nous étonnons cependant pas de trouver nombre de références à des réalités des colonies françaises d'alors : la majeure partie des realia du monde arabe trouvées dans le Littré à l'aide d'une recherche "musulman » ou "arabe » dans le texte complet du dictionnaire se retrouvent par exemple dans la nomenclature de Ghiotti (BAIRAIN, BÉDOUIN, BOURNOUS, CHÉRIF, CHÉRIFAT, DJINN, DONAR, GOUM, GOURBI, HAREM, MUEZZIN, MARABOU, RAMAZAN, ...). Cette insertion renforce en effet l'image d'un Empire français fort de ses possessions coloniales, celle de la «plus grande France » qui était aussi exaltée par Ferry en cette fin de siècle.

Une deuxième carence concerne une période historique qui semble peu intéresser Ghiotti comme nous le confirmait l'analyse des dates présentes dans son Vocabolario, principalement toute la période qui précède le XVII ${ }^{e}$ siècle. Me basant sur une recherche d'articles du Littré contenant « autrefois » (se référant non au signifiant mais au signifié) ou «moyen-âge », je n'ai trouvé que deux occurrences de mots de ce type présents dans les deux ouvrages: AUNEUR et BASOCHE, définis respectivement en italien 
par Ghiotti comme «ancien ministre sur les mesures » et «tribunal établi en 1302 par Philippe le Bel, pour juger des différends qui s'élevaient entre les clercs». Sont par contre absents, par exemple, BOUJONNEUR ${ }^{22}$, CAMOCAN $^{23}$, COLINETTE ${ }^{24}$, ESPRINGALEE ${ }^{25}$, ENÇOIR ${ }^{26}$, etc. On notera que l'intérêt de Ghiotti se porte dans le choix de ces mots sur des aspects de civilisation qui appartiennent à la sphère historico-politique plutôt qu'à la vie quotidienne des Français, et cette observation correspond en effet à ce qui ressort de l'analyse de l'ensemble des realia présentes dans le Vocabolario: les spécificités françaises dont Ghiotti se délecte dans son dictionnaire concernent avant tout l'homme de la fin du XVIII ${ }^{e}$ siècle et du XIX ${ }^{e}$ siècle, et bien peu son quotidien. Une recherche dans le texte entier du dictionnaire sur l'association «sorta di " (= sorte de) nous permet en effet de noter que l'on trouve nombre de mots se référant à des spécialités gastronomiques (BABA, CRÊPE, GALETTE, ...), des jeux (BIRIBI, BISTOQUET, BOUILLOTTE, BRELAN, BRISQUE, ...), des habits ou accessoires (INDISPENSABLE, JUSTAUCORPS, LÉVITE, ...) ou des danses (LOURE, MATELOTE, MENUET, ...) qui pourraient être considérés comme des realia, comme l'indique justement le fait que Ghiotti ne se contente pas de les traduire mais les définit aussi à l'aide d'un hyperonyme («type de jeu », «type de danse », ... ${ }^{27}$. Leur description s'arrête cependant à cette identification d'appartenance à une classe d'objets ou d'activités. Seuls quelques objets du quotidien ont droit à une glose plus détaillée dans le Vocabolario, mais ils le sont dans ce cas pour leur valeur symbolique par rapport aux us d'une période historique donnée. C'est le cas par exemple de CARMAGNole défini (en italien) comme "sorte d'air, de danse et de vêtement en vogue aux temps de la première Révolution française ", d'INCROYABLE "nom qui, en France, au temps du Directoire, était donné aux petits maitres, aux dandys et aux femmes élégantes qui s'habillaient avec une élégance exagérée et ridicule » ou de SERDEAU « Officier de bouche à la Cour, meuble et salle où mangeaient les gentilshommes de cour, lieu où l'on vendait les restes de table ». La plupart des realia qui donnent lieu à des gloses sont des mots et associations directement liés à la politique et à l'administration françaises, celles surtout de la période révolutionnaire et immédiatement postérieure. On trouve en particulier des appellatifs de personnes et de groupes de personnes, relatifs à des fonctions publiques ou politiques, comme par exemple ARCHITRÉSORIER ${ }^{28,}$ BLANCS ET BLEU ${ }^{29}$,

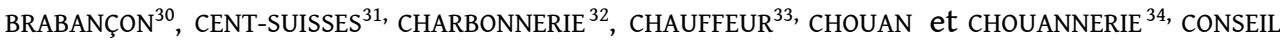
DES DIX, DES ANCIENS, DES CINQ-CENTS ${ }^{35}$, CONSTITUANTE ${ }^{36}$, etc. On trouve aussi des gloses dans les articles se référant à des édifices représentatifs d'événements liés à l'histoire de France $^{37}$, à des aspects de la vie citoyenne française dans lesquels ils sont reliés à des réformes (en particulier, révolutionnaires) : tous les mois du calendrier révolutionnaire sont présents mais aussi les divisions en départements, etc. Dans ces cas, Ghiotti ne manque pas de fournir des détails sur l'origine des objets décrits et leur relation à la période qu'ils représentent. Il glose la Marseillaise, par exemple, comme suit : « hymne patriotique et guerrier composé par Rouget de l'Isle (pron. $\mathrm{Hi}^{* 38}$ ) en 1792, par l'armée du Rhin $»^{39}$.

\section{Conclusion : le Vocabolario, un dictionnaire patriotique}

[...] ici la révolution n'est pas une affaire de race, mais d'intérêts et d'idées. Elle a commencé à la fin du siècle dernier, avec Beccaria par exemple, par la propagation de la littérature et de la philosophie françaises. C'est la classe moyenne, ce sont les gens éclairés qui la propagent, traînant le peuple après eux, comme jadis aux EtatsUnis pendant la guerre de l'indépendance. Il y a une force nouvelle, supérieure aux antipathies provinciales, inconnue il y a cent ans, située non dans les nerfs, le sang 
et les habitudes, mais dans la cervelle, les lectures et le raisonnement, d'une grandeur énorme, puisqu'elle a fait la révolution d'Amérique et la révolution française, d'une grandeur croissante, puisque les découvertes incessantes de l'esprit humain et les améliorations multipliées de la condition humaine contribuent chaque jour à l'augmenter. Suffira-t-elle à soutenir l'Italie? (Taine [1864] 1990 : 43-44).

Soutenir une Italie dont l'unification est terminée depuis peu, c'est une des missions de cette classe moyenne éclairée à laquelle appartient Ghiotti et de l'école nationale qu'il représente. Ce soutien trouve sa source dans la formule célèbre de Massimo D’Azeglio : «L'Italie est faite, il faut faire les Italiens »: pour faire les Italiens, il faut leur apprendre à se sentir membres d'une communauté nationale encore balbutiante, en exaltant non seulement la valeur du territoire et des institutions du sol national mais aussi celle d'autres communautés nationales dans le parcours qui a porté à leur définition.

L'inspecteur d'Académie détermine les limites de l'enseignement depuis l'histoire de la révolution française jusqu'à nous : d'autres voudraient que l'on dise de nous à la Révolution française par respect envers la didactique ; enfin l'ordre du jour est formulé, partagé par la majorité de l'assemblée tant pour ce qui concerne l'enseignement de l'histoire que celui de la géographie: histoire de la patrie destinée à former le caractère civil et moral de l'individu depuis la révolution française dans ses relations et influences en Italie, avec la méthode qui sera indiquée dans la discussion générale de l'enseignement objectif. Géographie particularisée dans le processus naturel du connu vers l'inconnu en commençant de l'habitation à l'école, de l'école à la ville pour élargir de la commune à la province et de celle-ci à l'Etat » (Conférences pédagogiques, année 1881 : Pérouse, Séance XI, 27 août 1881, cité par SANI $2003: 135$ [ma traduction] ${ }^{40}$ ).

Le Vocabolario comparativo, comme on l'a vu tout au long de la description de sa forme et de son contenu, s'inscrit parfaitement dans la lignée d'une rénovation des contenus et des méthodes d'enseignement de son époque. Il promeut en effet, par l'insertion d'une information "de civilisation", une vision du monde qui coïncide avec celle que véhiculaient les nouveaux programmes d'histoire et de géographie italiens et dans laquelle prédominent les modèles de la Révolution et de la I ${ }^{2}$ re République françaises. Pensé comme un instrument didactique, le dictionnaire de Ghiotti devient acteur dans la formation des nouvelles générations d'Italiens, son caractère bilingue permettant de mettre en comparaison tant les deux langues que les deux cultures qu'il décrit, dans le but de renforcer la connaissance des unes et des autres. 


\section{Sources primaires}

GHIOTTI, Candido (1868). Grammatica ragionata della lingua francese Nuovo corso di lezioni teorico-pratiche dettate col confronto della lingua italiana e coll'analisi filologica. Alexandrie : Tip. Ragazzone.

GHIOTTI, Candido (1875). Grammatica ragionata della lingua francese Nuovo corso di lezioni teorico-pratiche dettate col confronto della lingua italiana e con ragionamenti filologici. Turin : Petrini.

GHIOTTI, Candido (1884). Grammatica ragionata della lingua francese Nuovo corso di lezioni teorico-pratiche dettate col confronto della lingua italiana e con ragionamenti filologici. Rome : Paravia.

GHIOTTI, Candido (1890). Nuovo vocabolario comparativo delle lingue italiana e francese. Turin : Bellardi et Appiotti.

GHIOTTI, Candido (s.d.) [1889]. Vocabolario scolastico delle lingue italiana e francese Compendio del nuovo vocabolario comparativo delle lingue italiana e francese dello stesso autore. $10^{\mathrm{e}}$ édition Turin : Petrini.

\section{Sources secondaires}

BERNARD, Claude (1990). Comment nos ministres font l'histoire : le discours de l'instruction publique et ses procédés de persuasion. Toulouse : Presses Université du Mirail.

DE FRANCESCO, Antonino (2006). Mito e storiografia della 'grande rivoluzione'. La rivoluzione francese nel pensiero politico italiano del 900. Naples : Guida.

FARINA, Annick (2012). « Le traitement des mots d'une culture non partagée dans le dictionnaire bilingue : le lexique révolutionnaire dans les dictionnaires français-italien ». In Ligas, Pierluigi et Frassi, Paolo (éd.), Lexiques Identités Cultures. Vérone : QuiEdit, 281-302.

LILLO, Jacqueline (2006). « Les dictionnaires scolaires bilingues français/italien ». In Nadia Minerva (éd.). Lessicologia e lessicografia nella storia degli insegnamenti linguistici. Quaderni del CIRSIL 5. Bologne : CLUEB, 221-236.

MERGER, Marie-France (2003). « De Z à Z. Étude de quelques aspects caractéristiques du dictionnaire bilingue de Candido Ghiotti ». In Nadia Minerva (éd.). Lessicologia e lessicografia nella storia degli insegnamenti linguistici. Quaderni del CIRSIL 2. Bologne : CLUEB, 165-174. MERGER, Marie-France (2005). « Candido Ghiotti : une figure emblématique du professeur de français (Italie, fin XIX siècle) ». Documents pour l'histoire du français langue étrangère ou seconde, 35, 75-89.

MERGER, Marie-France (2007). « La place de l'énonciateur dans le dictionnaire scolaire bilingue de Candido Ghiotti ». In Nadia Minerva (éd.). Lessicologia e lessicografia nella storia degli insegnamenti linguistici. Quaderni del CIRSIL 5. Bologne : CLUEB, 165-174.

MERGER, Marie-France (2013). « Deux dictionnaires de Candido Ghiotti qui ont marqué le XIX ${ }^{\mathrm{e}}$ siècle ». In Jacqueline Lillo (éd.). Les best-sellers de la lexicographie franco-italienne $\mathrm{XVI}^{\mathrm{e}}-\mathrm{XXI}^{\mathrm{e}}$ siècle. Rome : Carocci, 115-132.

MESCHONNIC, Henri (1992). Des mots et des mondes - Dictionnaires, encyclopédies, grammaires, nomenclatures. Paris : Hatier. 
MINERVA, Nadia (2002). La règle et l'exemple À propos de quelques manuels du passé $\left(\mathrm{XVII}^{\mathrm{e}}-\mathrm{XX}{ }^{\mathrm{e}}\right.$ siècles). Bologne : CLUEB.

MINERVA, Nadia (2002). « France/Italie : une identité d'emprunt ou une identité en partage ?». Documents pour l'histoire du français langue étrangère ou seconde, 28, 119-134.

MINERVA, Nadia (2003). Insegnare il francese in Italia Repertorio di manuali pubblicati dal 1861 al 1922. Bologne : CLUEB.

PELLANDRA, Carla (1991). «Professeurs de français d'autrefois. Analyse de la revue La lingua francese nelle scuole secondarie d'Italia de 1883 à 1892 ». Documents pour l'histoire du français langue étrangère ou seconde, $8,221-228$.

PELLANDRA, Carla (2004). Le radici del nostro mestiere. Storia e storie degli insegnamenti linguistici. Quaderni del CIRSIL 3. Bologne : CLUEB.

PRUVOST, Jean (2009). « Quelques perspectives lexicographiques à mesurer à l'aune

lexiculturelle », Études de Linguistique Appliquée, 154, 137-154

PUREN, Christian (1985). « La motivation dans la méthode directe », Les Langues modernes, (« La motivation pour les langues »), 5, 69-77.

PUREN, Christian (1988). Histoire des méthodologies de l'enseignement des langues. Paris :

Nathan-CLE international.

SANI, Roberto \& TEDDE Angelino (2003). Maestri e istruzione popolare in Italia tra Otto e Novecento : interpretazioni, prospettive di ricerca, esperienze in Sardegna. Milan : Vita e Pensiero.

TAINE, Hippolyte (1990). D’Assise à Florence. Voyage en Italie II. Bruxelles : Editions Complexes.

\section{NOTES}

1. « dopo sette anni di lavoro paziente e di gravi fatiche» (Ghiotti $1890:$ V), c'est moi qui traduis.

2. « una matassa inestricabile».

3. Orthographe incorrecte

4. C'est une orthographe erronée

5. Mot d'utilisation commune mais pas italien

6. C'est un vilain mot à éviter

7. « Tu diras plutôt, mais tu diras plutôt, tu utiliseras de préférence ces mots, tu diras en bon italien ».

8. Pirouette

9. Bonnet

10. Buffet

11. Mot grossier

12. «Contribuiscono in seguito a rendere l'Opera migliore e più completa, relativamente alle esigenze delle nostre scuole secondarie e superiori : tutta la parte che si riferisce alla storia, alla mitologia, alla geografia, è stata intercalata, seguendo l'ordine alfabetico, nel testo stesso della nomenclatura generale delle due lingue e le notizie storiche, geografiche, statistiche che la illustrano; le spiegazioni relative ai termini tecnici di scienze, d'arti, di mestieri, di industrie; la pronuncia dei nomi proprii, quella delle voci di suono irregolare o dubbio o poco conosciuto ; le voci dei verbi irregolari ; il plurale dei nomi composti ; le osservazioni grammaticali relative al mutare di genere che fanno alcuni nomi, a seconda della loro accezione, ed infine la più larga parte fatta alla 
correzione dei veri francesismi e delle voci errate, accennando alle voci di buona lingua che possono, con non minore efficacia, e certamente con maggior purezza di elocuzione, sostituire queste e quelli ».

13. Merger voit dans cette pratique une caractéristique des "dictionnaires d'autrefois » mais je préfère l'attribuer au genre des «dictionnaires d'auteurs " parce qu'elle s'intègre dans des pratiques lexicographiques de différents types et époques (on la retrouve dans des dictionnaires d'aujourd'hui qui décrivent tant la «langue verte» que le bon usage, par exemple) mais n'apparaît jamais, à ma connaissance, dans des ouvrages conçus par des équipes.

14. Merger (2007 : 64) cite par ex. les commentaires de Ghiotti sur la marquise de Pompadour qui « exerça une influence néfaste sur les affaires du Gouvernement » ou sur Molière qui serait «le plus grand auteur comique français », Hugo étant pour sa part le "plus grand poète français du $\mathrm{XIX}^{\mathrm{e}}$ siècle » (les commentaires sont en italien dans le dictionnaire).

15. Nous n'avons pas pris en considération ici les choix de traduction des noms propres effectués par Ghiotti mais il serait intéressant de le faire parce que la proposition systématique de la forme traduite alors qu'elle entrait déjà en concurrence avec la forme originale dans les publications de son époque est probablement assimilable à une prise de position contre l'emprunt.

16. Par exemple « CALvados (pron. kalvadòss'), geog. Calvados (catena di scogli nella Manica, sulle coste della Normandia) - Calvados (geog.) Calvados (dipartimento della Francia, sulla Manica; capoluogo Caen : pron. kan)».

17. Citons l'information encyclopédique fournie sur Clarence qui se résume à : « frère d'Édouard IV, roi d'Angleterre, noyé dans un tonneau de malvoisie : 1478 » «Clarence (George, duc de), stor. Giorgio, duca di Clarence (fratello di Edoardo IV, re d'Inghilterra; annegato in una botte di malvasìa : 1478) »).

18. Pour mesurer la proportion des références aux différents pays (et surtout aux nationalités attribuées aux personnalités et lieux cités), et la grande présence de la France, on pourra comparer les résultats suivants : 600 occurrences de l'adjectif « francese » (dont près de 450 dans les articles relatifs à des noms propres), 28 de l'adjectif «italiano» et 78 de sa forme féminine « italiana » - dont 40 dans des commentaires «non è (buona) voce italiana » = ce n'est pas un (bon) mot italien, utilisés par Ghiotti pour définir des emplois italiens qu'il juge impropres - et enfin 20 de «spagnuolo » et 10 de « spagnuola».

19. Les pics de mortalité entre 1789 et 1793 ne sont évidemment pas dus à des causes naturelles mais aux années de la Terreur.

20. $C f$. en particulier Farina 2008 : pour cet article, j'avais recensé tous les mots particuliers à la période révolutionnaire qui apparaissaient dans les dictionnaires bilingues français-italien actuels, ce qui m'a permis une comparaison avec le contenu du dictionnaire de Ghiotti qui en recense de nombreux que l'on ne trouve plus dans les nomenclatures de dictionnaires de petites dimensions et qui propose surtout des équivalents italiens pour ces mots que les dictionnaires actuels ne fournissent pas, se contentant généralement de les gloser.

21. C'est le cas par exemple des quelques vins français qui apparaissent dans le dictionnaire (Bordeaux, Bourgogne, Champagne, ...) : quoique provenant d'une région particulière, ils sont bus par tous les Français.

22. «Se disait autrefois d'un juré du corps des drapiers » (Littré).

23. « Sorte d'étoffe précieuse usitée dans le moyen âge " (Littré).

24. «Sorte de bonnet que les femmes portaient autrefois en déshabillé » (Littré).

25. « Espèce de baliste autrefois en usage dans les armées du moyen âge » (Littré).

26. « Sorte de sinet usité autrefois dans les livres d'église » (Littré).

27. Par exemple, « BIRIBI sm. biribisso (sorta di giuoco a carte) » ou « CRÊPES sf.pl. (t. di cuc.) galletti (sorta di frittelle)». Dans le deuxième exemple, Ghiotti démontre sa piètre connaissance de la gastronomie française ou de la gastronomie tout court qui est sûrement à l'origine de ce que l'on peut considérer comme une erreur due à des «faux-amis ». Littré définit ainsi le mot crêpe : 
« CRÊPE nf (krê-p') 1 Aliment commun dans l'ouest de la France où il est fait avec de la farine de blé noir détrempée, puis cuite en l'étendant par couches minces sur une poêle destinée à cet usage. Crêpe beurrée. Crêpe sèche. 2 Sorte de petite galette faite avec la farine de froment, à laquelle on joint souvent du sucre, des œufs et quelque aromate et que l'on fait cuire à la poêle avec un tout petit morceau de beurre ou de graisse ». Ghiotti ne s'occupe pas du premier sens (probablement parce qu'il est indiqué comme relatif à la gastronomie bretonne) mais il traduit l'hyperonyme " galette » utilisé par Littré pour le deuxième sens par « galletto » dont le seul sens culinaire est défini dans le dictionnaire de la Crusca ( $5^{\mathrm{e}}$ édition 1863-1923) comme « dicesi a una Specie di pasta fritta, che ha figura simile a quella della creta del galletto». En italien, le " galetto » se référait donc à une pâte frite (en forme de crête de coq), ce qui explique la glose « sorte de beignet » qui suit l'utilisation de ce traduisant dans l'article « crêpes » de Ghiotti. Or, on le sait, la crêpe n'est pas plus une pâte frite en forme de crête de coq qu'une sorte de beignet.

28. « ARCHITRÉSORIER sm. ( $t$. di stor.) arcitesoriere (gran tesoriere al tempo del $1^{\circ}$ Impero francese)».

29. « BLANCS ET BLEU* (stor.) Bianchi e Turchini (nomi dati durante le guerre della Rivoluzione francese, il primo ai monarchici, partitenti del vessillo bianco; il secondo ai soldati della Repubblica)».

30. « BRABANÇONS sm. pl. ( $t$. di stor.) avventurieri allemanni e fiamminghi che nel medio evo saccheggiavano la Francia ».

31. « CENT-SUISSES sm.pl. i Cento Svizzeri (soldati svizzeri che, dal 1471 sino al 1830, servirono come di guardaportone, guardie d'onore, ai re di Francia)».

32. « CHARBONNERIE $s f$. ( $t$. stor.) Carboneria (società segreta formatasi in Francia al tempo della Restaurazione ; 1815) ».

33. « CHAUfFEUR sm. [...] (al pl., t. stor. franc.) nome di certi briganti al tempo della guerra fra la Vandea ed i repubblicani francesi ».

34. « CHOUAN sm. (t. stor.) insorto della Vandea (ai tempi della prima Rivoluzione francese e dopo il 1830) »; « Chouannerie sf. guerra di insorti, di partigiani ; il partito degli insorti della Vandea ».

35. « CONSEIL DES DIX (stor.) Consiglio dei Dieci (tribunale segreto e terribile della repubblica di Venezia), stabilito nel 1310 ; - Conseil des Anciens et Conseil des Cinq-Cents (stor.) Consiglio degli Anziani e Consiglio dei Cinquecento (nome di due assemblee politiche istituite nell'anno terzo della prima Repubblica francese) ».

36. « CONSTITUANTE (l'Assemblea nazionale di Francia che diede la Costituzione del 1791)».

37. «BASTILLE (stor.) Bastiglia (castello a Parigi, costruito sotto i regni di Carlo V e di Carlo VI, e demolito dal popolo nel 1789) »; « CHÂTELET sm [...] (t. di stor. franc.) il Châtelet (luogo che serviva di tribunale e di prigione in Parigi)»; etc.

38. L'astérisque indique que la lettre h est ici 'aspirée'.

39. " MARSEILLAISE (LA), sf. (t. stor.) la Marsigliese (inno patriottico e guerriero composto da Rouget de l'Isle (pron. $\left.\mathrm{HI}^{*}\right)$, nel 1792, per l'esercito del Reno)».

40. «Il R. Provveditore determina i limiti dell'insegnamento storico dalla rivoluzione francese a noi : altri vorrebbe si dicesse da noi alla rivoluzione francese per ossequio alla didattica; finalmente si formola il seguente ordine del giorno che accontenta la maggioranza della assemblea sia riguardo all'insegnamento della storia sia a quello della geografia : Storia patria principalmente intesa a formare il carattere civile e morale dell'individuo dalla rivoluzione francese nelle sue relazioni ed influenza in Italia, con quel metodo che sarà indicato nella discussione generale dell'insegnamento oggettivo. Geografia particolareggiata con processo naturale dal noto all'ignoto cominciando dall'abitazione alla scuola dalla scuola alla città ed allargandosi dal comune alla provincia e da questa allo Stato » (Conferenze pedagogiche, anno 1881 : Perugia, Seduta XI, 27 agosto 1881). 


\section{RÉSUMÉS}

Nous intéressant à la manière dont la diffusion de la culture de l'autre a pu accompagner l'enseignement des langues étrangères et en particulier à la fonction qu'on a pu donner au dictionnaire bilingue dans cette diffusion, nous avons examiné un ouvrage qui peut être considéré comme pionnier dans l'adaptation de la forme et du contenu du dictionnaire bilingue à des préoccupations didactiques, dans le contexte de l'enseignement du français langue étrangère en Italie : le Nuovo vocabolario comparativo delle lingue italiana e francese de Candido Ghiotti (1883). Marie-France Merger illustre l'adaptation de la microstructure de ce dictionnaire à des besoins didactiques linguistiques spécifiques, par l'illustration des différences entre les deux systèmes décrits (français vs italien). Annick Farina s'attache pour sa part à démontrer combien cette volonté d'instruire s'applique aussi à des aspects concernant les cultures véhiculées par ces langues et combien le Vocabolario entre dans un projet plus large de diffusion d'une vision de la civilisation française édifiée en modèle dans l'Italie post-unitaire.

Given our interest in the ways in which the dissemination of the culture of others can be an aid in the teaching of foreign languages and in particular in the function we can give to a bilingual dictionary in such dissemination, we have examined a book that can be considered a pioneer work in its adapting the form and content of the bilingual dictionary for educational purposes in the context of teaching French as a Foreign Language in Italy: the Nuovo vocabolario comparativo delle lingue italiana e francese by Candido Ghiotti (1883). Marie-France Merger illustrates Ghiotti's adaptation of the dictionary's microstructure to specific language learning needs by illustrating the differences between the two described systems (French vs. Italian). Annick Farina demonstrates how this desire to instruct also applies to aspects concerning the cultures conveyed by these languages and how the Vocabolario becomes part of a larger project for disseminating a view of French civilization as a model to be imitated in post-Unification Italy.

\section{INDEX}

Keywords : bilingual lexicography, Candido Ghiotti, teaching of French as a foreign language, civilization, realia

Mots-clés : lexicographie bilingue, Candido Ghiotti, didactique du FLE, civilisation, realia

\section{AUTEURS}

\section{ANNICK FARINA}

Université de Florenceannick.farina@unifi.it

\section{MARIE-FRANCE MERGER}

Université de Pisemarie.france.merger@unipi.it 